\title{
From microwatt to gigabit: challenges of modern radio design
}

\author{
H. Pretl IEEE, OVE, T. Faseth IEEE, T. Schumacher IEEE, M. Stadelmayer IEEE, S. Sadjina IEEE, S. Schmickl IEEE, \\ E. Hager IEEE
}

On the brink of introducing the fifth generation (5G) of cellular networks, the art of radio-frequency (RF) integrated circuit design has never seen such a wide spread of diverging requirements:

On the one hand, ubiquitous sensor networks are mandating power budgets in the order of micro-watt. They should be constructed as energy-autonomous, wireless, low-cost sensor nodes. This demand is caused by massive deployment scenarios of billions of devices, which makes wires and batteries unpractical. As a result, the desire for the radio nodes to harvest their operational energy from the environment emerges.

On the other hand, the recent and ongoing realization of gigabit-per-second capable cellular modems is driving hardware and power requirements to extremes. To overcome hardware limitations, introduced by analog impairments, digital correction and alignment algorithms are employed for compensation. These factors call for usage of expensive advanced CMOS technology nodes and increased utilization of digital signal processing techniques.

In this paper, we recap ongoing trends and developments for ultra-low power and high-end transceiver (TRX) designs using CMOS technology nodes ranging from low-cost to highest performance.

Keywords: RF; ultra-low-power; wireless architectures; transceiver; mixed-signal circuits; $5 \mathrm{G}$

\section{Von Mikrowatt zu Gigabit: Herausforderungen beim Design moderner Funkübertragungssysteme.}

Neben der Einführung der zellularen Mobilfunknetze der fünften Generation (5G) muss sich auch die integrierte HochfrequenzSchaltungstechnik einer noch nie dagewesenen Breite an unterschiedlichen Anforderungen stellen:

Auf der einen Seite gibt es überall kabellose Sensornetzwerke, denen nur eine geringe Menge an Versorgungsleistung im MikrowattBereich zur Verfügung steht. Daher müssen die Chips in diesem Bereich energieautark und möglichst kostengünstig entwickelt werden. Aufgrund der zu erwartenden hohen Stückzahlen (Milliarden von unterschiedlichsten Sensoren für verschiedene Anwendungen) sind eine drahtgebundene Energieversorgung oder der Einsatz von Batterien in vielen Fällen praktisch unmöglich. Somit wird es für die Sensoren immer wichtiger, ihre Energieversorgung aus der Umgebung zu gewinnen (Stichwort Energy Harvesting). Auf der anderen Seite treibt die moderne Funkkommunikation in 5G-Netzwerken mit Gigabit-pro-Sekunde-fähigen zellularen Modems die Hardwareund Leistungsverbrauchsanforderungen auf die Spitze. Zur Verbesserung hardwarebedingter Einschränkungen und Ungenauigkeiten durch analoge Realisierungen werden nun oft digitale Korrektur-, Anpassungs- und Kompensationsalgorithmen eingesetzt. Diese neuartigen Mixed-signal-Schaltungskonzepte sowie die erforderliche gesteigerte Integrationsdichte verlangen oft auch den Einsatz von teuren immer kleiner werdenden CMOS-Technologieknoten.

In dieser Arbeit stellen die Autoren die aktuellen Trends und Entwicklungen in den Bereichen kostengünstiger Ultra-Low-Power- und High-End-Hochfrequenz integrierte CMOS-Schaltungen gegenüber.

Schlüsse/wörter: Hochfrequenz (HF); extrem niedriger Stromverbrauch; drahtlose Architekturen; Sende-Empfangsgeräte; Mixed-Signal-Schaltkreise; $5 \mathrm{G}$

Received August 31, 2017, accepted November 26, 2017, published online January 10, 2018 (C) The Author(s) 2018. This article is published with open access at Springerlink.com

\section{Introduction}

Taking a look at the recent forecast on mobile communication from the Ericsson mobility report [10] the number of mobile phone subscriptions should experience a small growth rate of approximately $3 \%$ per year, compared to an annual growth of roughly $20 \%$ for internet-of-things (IOT) devices, as visualized in Fig. 1(a). Simultaneously the total traffic for mobile phones, shown in Fig. 1(b), is expected to increase by a yearly rate of more than $40 \%$. This illustrates the tendency towards highest performance data services in $5 \mathrm{G}$ networks with Gbps range data rates to increase average speed per user as well as network capacity. To achieve such performance data, novel circuit design strategies are needed and hardware limitations need to be compensated by digital correction and alignment
Pretl, Harald, Johannes Kepler University Linz, Energy-Efficient Analog Circuits \& Systems, Altenberger Straße 69, 4040 Linz, Austria; DMCE GmbH \& Co KG, a subsidiary of Intel Corp., Freistädterstraße 400, 4040 Linz, Austria (E-mail: harald.pretl@jku.at); Faseth, Thomas, Johannes Kepler University Linz, Energy-Efficient Analog Circuits \& Systems, Altenberger Straße 69, 4040 Linz, Austria (E-mail: thomas.faseth@jku.at); Schumacher, Tim, Johannes Kepler University Linz, Energy-Efficient Analog Circuits \& Systems, Altenberger Straße 69, 4040 Linz, Austria (E-mail: tim.schumacher@jku.at); Stadelmayer, Markus, Johannes Kepler University Linz, Energy-Efficient Analog Circuits \& Systems, Altenberger Straße 69, 4040 Linz, Austria

(E-mail: markus.stadelmayer@jku.at); Sadjina, Silvester, Johannes Kepler University Linz, Energy-Efficient Analog Circuits \& Systems, Altenberger Straße 69, 4040 Linz, Austria; DMCE GmbH \& Co KG, a subsidiary of Intel Corp., Freistädterstraße 400, 4040 Linz, Austria (E-mail: silvester.sadjina@intel.com); Schmickl, Stefan, Johannes Kepler University Linz, Energy-Efficient Analog Circuits \& Systems, Altenberger Straße 69, 4040 Linz, Austria (E-mail: stefan.schmick|@jku.at); Hager, Ehrentraud, Christian Doppler Laboratory for Digitally Assisted RF Transceivers for Future Mobile Communications, Johannes Kepler University Linz, Altenbergerstraße 69, 4040 Linz, Austria (E-mail: ehrentraud.hager@jku.at); Johannes Kepler University Linz, Energy-Efficient Analog Circuits \& Systems, Altenberger Straße 69, 4040 Linz, Austria 


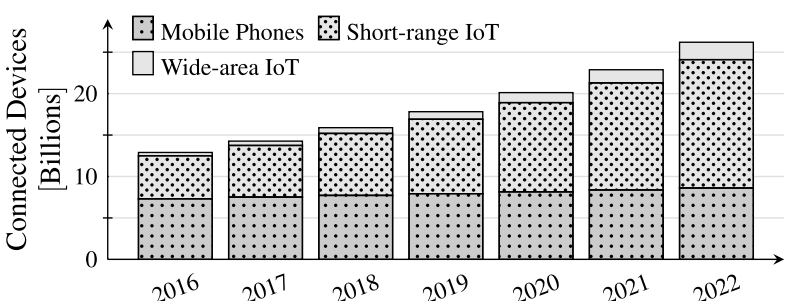

(a)

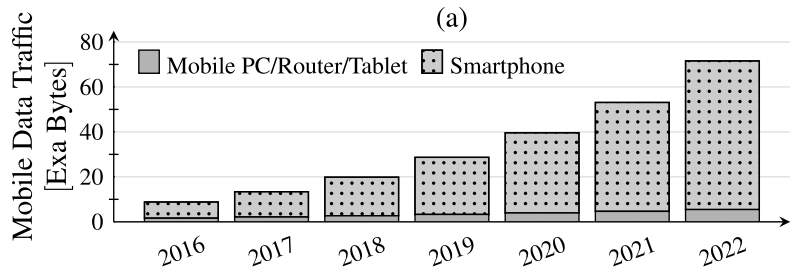

(b)

Fig. 1. Summary of Ericsson Mobility Report [10]

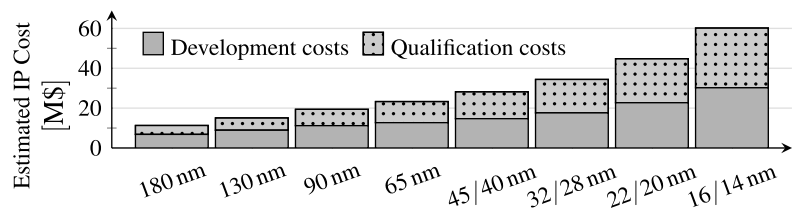

Fig. 2. Perspective on IP developing and qualification cost in different technology nodes [16]

algorithms. Often the most expensive and advanced CMOS technology nodes, i.e. $14 \mathrm{~nm}$ FinFET [17], are used, since they imply best performance and highest integration level for the integrated circuits while still maintaining decently low power consumption. Figure 2 shows a perspective on the costs of development and verification for intellectual properties (IPs) in different technologies as outlined in [16]. With a large production volume for the potential application, the enormous development and fabrication costs of those extraordinary small technology nodes can be justified. Furthermore, these technologies allow for advanced high speed digital signal processing (DSP) techniques. Section 3 will recap ongoing developments and impairments in high performance mobile transceivers.

With a predicted number of more than fifteen billion short-range loT-devices in 2022, power supply will be a certain issue. Since powering billions of devices using batteries is impractical for ecological and economical reasons, it is desirable that loT-devices harvest their operational energy from their environment. Therefore, the associated transceiver design is discussed and must incorporate power efficiency measures in order to get along with the limited amount of energy available from the energy-harvesting techniques.

At the same time loT-devices should cover a broad range of applications, including specialized implementations with relatively low quantities to be manufactured, e.g. for specialized medical applications or environmental monitoring, and simultaneously provide lowest product cost. As a reasonable consequence to fulfill these requirements, mature CMOS technology nodes (like $130 \mathrm{~nm}$ or $180 \mathrm{~nm}$ ) are required instead of using the most advanced technologies.

Therefore, in Sect. 2.1, we recap recent publications and common energy-reduction techniques for ultra-low-power (ULP) transceiver design in $180 \mathrm{~nm}$ technologies for the frequency band at around 868-915 MHz since the corresponding wavelength offers a good

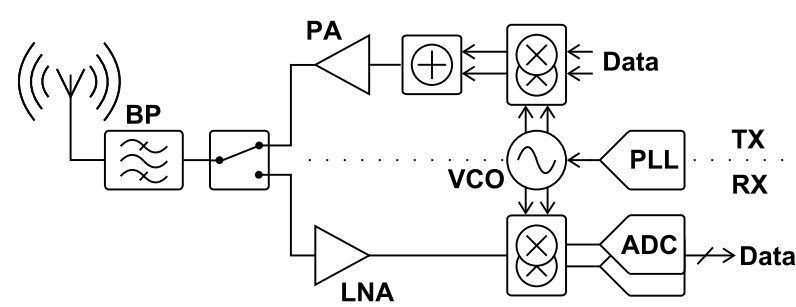

(a)

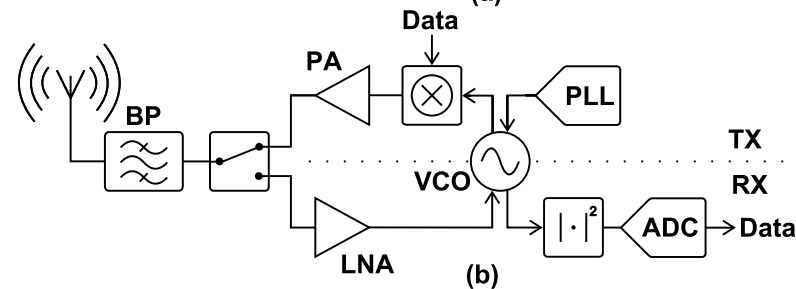

(b)

Fig. 3. Generic transceiver architectures for time-division-duplexing (TDD): (a) zero-IF architecture, and (b) ultra-low-power architecture

compromise between maximum-range of operation and antennasize [34]. In Sect. 2.2 various approaches of energy harvesting are summarized and available power budgets discussed.

In Sect. 3 we summarize ongoing trends in $5 G$ wireless communication and present the utilized most advanced CMOS technology nodes and discuss the corresponding circuit design challenges caused by the strong scaling of circuit structures. Finally, Sect. 4 summarizes and concludes the paper.

\section{Ultra-low-power transceiver design and energy harvesting}

The growing loT market demands a large amount of self-powered devices with long-term deployment. In order to increase the lifespan of those devices for a given energy source, the power consumption of every single component has to be minimized. A summary of the state-of-the-art can be found in [29]. Especially the wireless data transmission of such an loT sensor node consumes a lot of power and thus needs to be thoroughly optimized. Furthermore, an approach to extend the lifetime of a device is to harvest energy from the environment - either instead of or in addition to the battery.

Consequently, the following section proposes methods to design power saving ultra-low-power (ULP) transceivers and afterwards recaps some recent energy harvesting concepts.

\subsection{Energy-efficient transceiver architectures}

The typical approach to design transceivers for high performance applications is to utilize zero- or low intermediate frequency (IF) IQ-based architectures as shown in Fig. 3(a) [19]. This architecture transmits data by applying spectrally efficient complex modulation and demodulation schemes like Quadrature-Amplitude-Modulation (QAM) or Phase-Shift-Keying (PSK). However, the structure suffers from a high power consumption due to the dual modulation paths and the generation of the local oscillator signals. Thus, in order to save power, it is reasonable to reduce the amount of energy hungry components to a minimum while also reducing the overall systems complexity. For this reason, Frequency-Shift-Keying (FSK) or Amplitude-Shift-Keying (ASK) are used because they typically omit IQ-modulation/demodulation paths by using envelope detection for ASK for example. Figure 3(b) pictures a generic low-complexity transceiver structure.

Depending on the application, ULP transmitters generate the radio frequency $(\mathrm{RF})$ using a phase-locked-loop (PLL) and directly 
transmit the modulated signal. Such transmitter architectures can be summarized as PLL-based transmitters [15], and are often used in Bluetooth Low Energy (BLE) and Wireless Body Area Network (WBAN) applications [6]. PLL-based transmitters provide low phase noise and good frequency stability at the cost of increased power consumption. Nevertheless, in order to further reduce power, the PLL in the transmitter path can either be replaced by a high-quality (Q) oscillator, directly resonating at RF [9] or IF [26], or can fully be omitted by using a free running oscillator $[3,8]$ at the cost of reduced frequency- and phase-stability, e.g. caused by temperature, supply voltage, or process variations. Consequently, for this type of transmitter, modulation schemes like On-Off-Keying (OOK) are used since they do not require good frequency accuracy. However, the modulated signals have to fulfill the applicable local authority and spectral regulations which can be challenging.

The proposed generic structure in Fig. 3(b) shows a voltage controlled oscillator (VCO) which is shared among transmitter and receiver. During receive times the signal is injected into the VCO causing the oscillation to lock to a certain frequency and simultaneously modulating the amplitude [27]. This results in an amplification of the received signal dependent on the quality of the LC-tank within the VCO. This concept is used in the so called injection-locked [3] or Q-enhanced [8] receivers. In order to improve sensitivity, the generic structure is easily modified to make use of the super-regenerative concept, originally proposed by Armstrong [2]. If the desired communication range is short, the receiver can further be simplified by omitting the injection-step and directly demodulate the envelope as demonstrated in [18].

In addition to the energy-efficient architectures for each building block of the overall system, the overall power consumption can further be reduced by implementing duty-cycling or wake-up structures. During duty-cycling, the transceiver operation is separated in active and inactive states, defined by the duty-cycling ratio. This method allows to trade in the maximum achievable data rate for reduced power consumption [5]. Typically, wake-up structures are implemented using always-on diode-detector based receivers in order to detect a specified low-rate wake-up sequence. Correlation methods can be used to improve the sensitivity of the additional wake-up receiver [24].

\subsection{Energy harvesting}

There are two popular possibilities to harvest energy from the environment, using either incidental electromagnetic (EM) waves in the $\mathrm{MHz}$ - up to $\mathrm{GHz}$-range or ambient light. ${ }^{1}$ A general drawback is that the available spectral power density is quite low. For example, the transmitter of the potential energy source could be located several kilometers away from the energy harvesting system itself. Because of the strong decay of the EM far-field, the RF output power of the transmitter should often be in the kilo-watts region, which is usually best fulfilled by digital TV broadcast stations. As experimentally demonstrated in downtown Tokyo, a micro-controller was powered wirelessly by 9 TV channels in the frequency range of 512-566 MHz of a local TV broadcast station $6.3 \mathrm{~km}$ away [32]. The measured average harvested $D C$-power of the system was $\approx 15-17 \mu \mathrm{W}$ at $4.1 \mathrm{~V}$, whereby the system consisted of a relatively large log-periodic antenna, a discrete RF charge-pump, and the PIC24F micro-controller. Currently, similar experiments are carried out for energy harvesting from LTE mobile communication links [25].

${ }^{1}$ Of course, for some applications piezoelectric generators using force or vibration as well as thermoelectric generators can be used effectively.
Besides harvesting generally available ambient RF-energy, it is also possible to use a local RF-power-transmitter to energize several surrounding mobile nodes in a range of a few meters. For example, the frequency band at the UHF ISM band 902-928 MHz can be utilized where the propagation losses are lower compared to higher frequencies. Dependent on the local regulations, the maximum allowed transmitted power in this band amounts to $36 \mathrm{dBm}$ EIRP, which corresponds to a maximum available power of $27 \mu \mathrm{W}$ at a distance of $10 \mathrm{~m}$ [21]. So the circuit design challenge is to build an RF to DC rectifier circuit with highest possible efficiency, in the best case of over $40 \%$, where a threshold voltage compensation circuit can effectively maximize the efficiency and decrease the input sensitivity at the same time [36]

Another possibility to harvest energy from the environment is the integration of photo-voltaic (PV) power (solar) cells into a standard CMOS-process. Thus, by realizing the PV cell on chip, a single-IC solution for energy supply and application specific circuitry can be provided. A major advantage of this method is the decreased number of components involved in the overall system and the compact realization. In order to overcome the permanent dependence on ambient light, the harvested energy can be stored on a capacitor. Size and design of the solar cell determine the generated power on the chip.

PV solar cells can either be integrated by post-CMOS processing as shown in e.g. [22, 23], or directly into a standard CMOS technology as proposed in e.g. [14]. In comparison to standalone PV cells, which achieve an energy conversion factor around 24\% [33], integrated standard-CMOS PV cells are limited to $\approx 2 \%$ efficiency [20, $30,31]$. The latter provide an output power of about $80 \mu \mathrm{W} / \mathrm{mm}^{2}$ at an illumination level of 25 klux (equivalent to a sunny, clear day in winter) and $1.4 \mu \mathrm{W} / \mathrm{mm}^{2}$ at 435 lux (equivalent to typical office illumination level).

\section{Next generation wireless architectures}

As presented in Sect. 1, the ever-increasing market penetration of smart-phones and mobile data usage rises to levels almost unthinkable a few years ago. In the following section the basics for the $5 \mathrm{G}$ wireless technologies and corresponding challenges for circuit design will be summarized.

\subsection{LTE and 5G basics}

From communications theory (Shannon's law) follows a sufficient signal-to-noise ratio (SNR) in conjunction with the inherent use of higher-order modulation schemes, or a dramatically increased transmission bandwidth is needed to cope with raised data transmission rates. Thus, the third generation partnership project (3GPP) is currently enhancing the fourth generation long term evolution (LTE) framework by several staged releases over the next months, with recently releasing the $5 \mathrm{G}$ New Radio (NR) architecture [1]. LTEAdvanced in the 3 GPP release 13 and beyond uses a multitude of often disjunctive frequency bands, higher-order modulation schemes like 256QAM, $2 \times 2$ or $4 \times 4$ multiple-input-multiple-output (MIMO), and link aggregation in time division duplex (TDD) and frequencydivision duplex (FDD) modes. In 5G NR, LTE and its techniques will serve as anchor nodes to finally realize true multi-Gbps wireless data transmission rates.

Especially the frequency bands below $3 \mathrm{GHz}$ are highly populated and fragmented due to the governmental licensing to several mobile operators within those bands. Consequently, to enlarge transmission bandwidth, LTE-Advanced defines three types of carrier (link) aggregation (CA) scenarios. As depicted for a simplified two channel aggregation concept in Fig. 4, there is a mode where neigh- 
boring carrier components (CC) - which are located within one frequency band - are combined to an intra-band contiguous mode, or non-neighboring channels form a non-contiguous CA channel. In LTE-Advanced, up to 5 channels in different frequency bands can be aggregated, each consisting of up 4 CC resulting in a maximum of $80 \mathrm{MHz}$ bandwidth per channel. In contrast to backwards compatible LTE, $5 G$ NR is also intended to flexibly utilize licensed and unlicensed bands up to millimeter wave (mm-wave) center frequencies. Additionally, the used bandwidths will rise up to $800 \mathrm{MHz}$ or above.

From the perspective of the RF front-end and overall system design, especially the simultaneous transmission and reception of several aggregated large bandwidth channels introduces numerous challenges to wireless RF integrated transceivers. The use of multiple transmit (TX) or receive (RX) frequency bands in conjunction with up to $8 \times 8 \mathrm{MIMO}$ antenna configurations increases the need

(a)

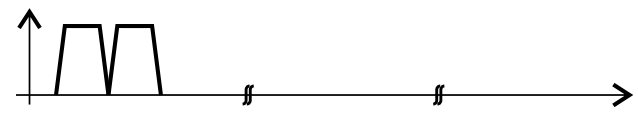

(b)

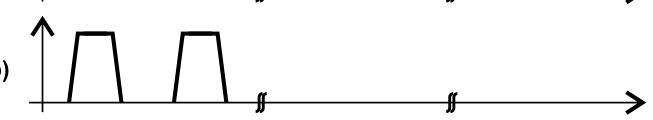

(c)

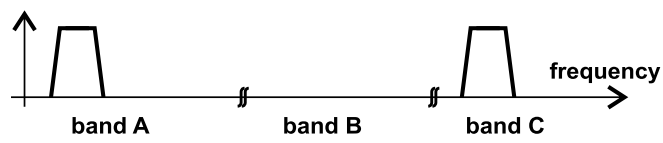

Fig. 4. Carrier Aggregation (CA) modes: (a) intra-band contiguous $C A$, (b) intra-band non-contiguous $C A$, and (c) inter-band $C A$ for scaling of the structures within the integrated circuits for performance, power, and chip-size reasons. This inherently justifies the use of the smallest and thus most expensive technology nodes available (cf. Fig. 2). Nevertheless, also coupling effects and their obvious negative consequences are increasingly challenging, even if proper circuit design and layout techniques are used.

\subsection{Simplified LTE-advanced transceiver architecture}

Like other wireless transmitters, also LTE-Advanced transmitters use distinct receive and transmit paths. Figure 5 depicts a simplified block diagram of such a multi-band integrated architecture utilizing two TX and two RX paths, but neglects further MIMO or auXiliary RX or TX paths. Nevertheless, still within this simplified block diagram, a general flexibility of configuration and adaption to different CA modes of operation is maintained. Of course, additional switches and multiplexers would be needed for true multi-band operation e.g. to configure the structure to operate in TDD mode using one PLL for TX and RX simultaneously keeping in mind that all these additional switching-paths can introduce additional unwanted coupling across the functional blocks. In contrast to Sect. 2.1, where there is a large spread of topologies and architectures, these high performance receivers and transmitters almost all utilize either zeroor low-IF topologies followed by high dynamic range (DR) analogdigital-converters $(A D C)$ or digital-analog-converters (DAC) and extensive digital signal processing in the baseband (BB). These days, for example, mixed signal cancellation for carrier aggregation LTE receivers as the one depicted in Fig. 6 [28], use novel circuitry to sense for modulated interference and generate an interference cancellation signal in the digital domain to subsequently compensate for analog impairments and coupling effects which are generally often

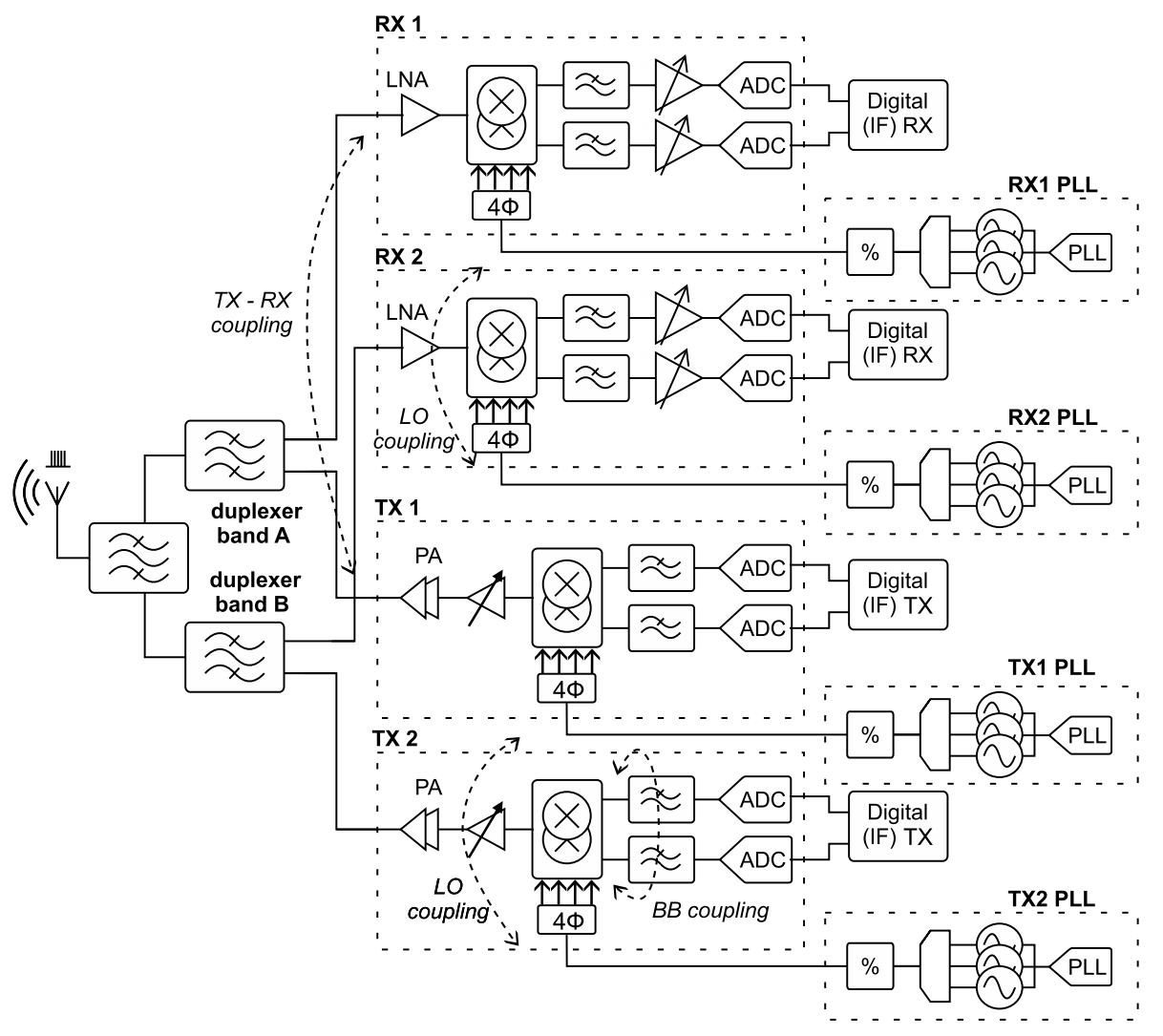

Fig. 5. Simplified inter-band CA transceiver structure 


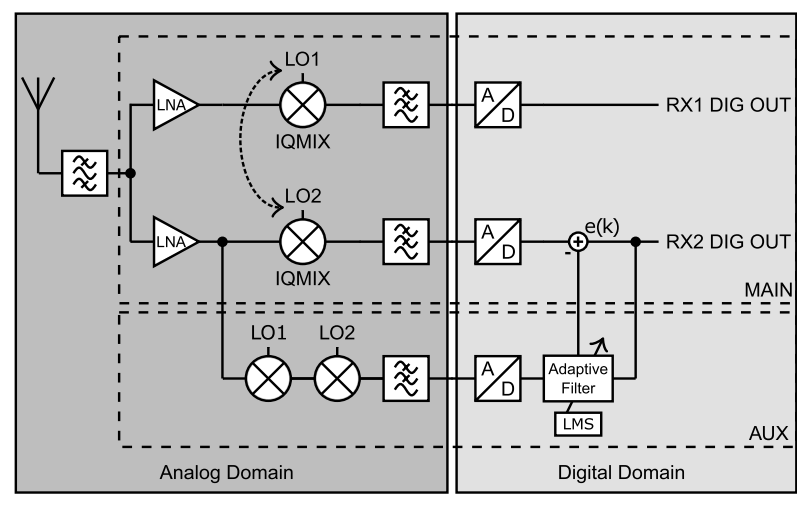

Fig. 6. Mixed signal CA LTE receiver architecture for the cancellation of modulated spurs [28]

summarized as self-interference effects and corresponding mitigation techniques.

Figure 5 summarizes some of those effects. Besides classical selfinterference problems, like $T X$-to- $R X$ coupling due to limited amount of isolation in a duplexer filter in FDD mode, also local oscillator (LO) pulling by the TX power amplifier (PA) or PA non-linearities, TX harmonics, or oscillator phase noise, and a combination of all those in and in-between several components of the system will lead to a significant desensitization of the corresponding receive paths. Also non-ideal BB signals (non-orthogonality and IQ coupling), here summarized as $B B$ coupling, degrade the error vector magnitude (EVM) and adjacent channel suppression. Of course, also LO coupling, which can also be harmonically related between multiple RX and TX bands in CA scenarios and corresponding phase noise must be considered. All these effects are consequently reducing the signalto-noise or -interference ratio. As self-interference poses a critical issue in CA-enabled TRX, many researchers and companies are trying to resolve the coupling effects within the IC to enhance the performance of their implementations. Foremost, analog mitigation techniques like fully-differential symmetrical structures or on-chip isolation techniques are used. But, as the results are often insufficient in terms of performance for the large number band combinations and scenarios, further adaptive digital signal processing is used to compensate for those coupling effects $[13,35]$.

Currently, most of the manufacturers of mobile communication chip-sets realize their RF TRX designs in $40 \mathrm{~nm}$ [7], $28 \mathrm{~nm}[11,12]$, or similar CMOS technologies.

Recently, the latest designs are fabricated in $16 \mathrm{~nm}$ or $14 \mathrm{~nm}$ FinFET CMOS $[4,17]$. The corresponding advantages of low voltage operation as well as drastically increased transit frequency and integration density are utilized for the realization of novel circuit design concepts, e.g. in [4] where a specialized type of filter-less reconfigurable harmonic rejection mixer has been shown or [17] where a ultra-low power wide-band RX circuit for CA scenarios was demonstrated.

\section{Conclusion}

This paper summarizes the ongoing - often conflicting - approaches in state-of-the-art (SoA) integrated circuit design for communication systems. By weighing the factors IP development and production costs, integration level, energy efficiency, the potential production volume of a realized product, and driven by the needs of the corresponding applications, the designers of integrated circuits either select well-established cost efficient technologies as $180 \mathrm{~nm}$ CMOS or costly leading edge technologies like $14 \mathrm{~nm}$ FinFET for their designs. For both cases, industry as well as academia researchers face a widespread field of unexplored challenging topics for integrated circuit and system design.

Based on two examples of wireless transmission systems, we present our current research focus. On the one hand, we work on ultra-low power RF transceivers for loT and health-care applications. Here we want to employ SoA mixed-signal circuit techniques for efficient signal generation and further enhance the sensitivity and interference immunity by adopting the technique of translational circuit techniques to the RF domain. On the other hand, due to the clear tendency to highest speed and lowest power wireless data transfer in $4 G$ and $5 G$ applications, RF integrated circuit designers face entirely new problem statements to be tackled using the most advanced technologies available. Due to impairments caused by the required massive parallelization in terms of multi-band and multi-antenna RF paths as well as signal bandwidths of multiples of $100 \mathrm{MHz}$, we work on novel circuit topologies in analog and digital domain to sense for non-idealities and coupling artifacts typical for such systems.

\section{Acknowledgements}

Open access funding provided by Johannes Kepler University Linz. The authors wish to acknowledge DMCE GmbH \& Co KG, a subsidiary of Intel Corp., for supporting this work partly carried out at the Christian Doppler Laboratory for Digitally Assisted RF Transceivers for Future Mobile Communications. The financial support by the Austrian Federal Ministry of Science, Research and Economy and the National Foundation for Research, Technology and Development is gratefully acknowledged.

Open Access This article is distributed under the terms of the Creative Commons Attribution 4.0 International License (http://creativecommons.org/ licenses/by/4.0/), which permits unrestricted use, distribution, and reproduction in any medium, provided you give appropriate credit to the original author(s) and the source, provide a link to the Creative Commons license, and indicate if changes were made.

\section{References}

1. 3GPP (2016): 3GPP LTE framework. Release 15. http://www.3gpp.org/release-15. Seen August 23. 2017.

2. Armstrong, E. H. (1922): Some recent developments of regenerative circuits. Proc. IRE, 10(4), 244-260.

3. Bae, J., Yan, L., Yoo, H. J. (2011): A low energy injection-locked FSK transceiver with frequency-to-amplitude conversion for body sensor applications. IEEE J. Solid-State Circuits, 46(4), 928-937

4. Bhagavatula, V., Kwon, D., Lee, J., Bui, Q. D., et al. (2017): 13.3 a SAW-less reconfigurable multimode transmitter with a voltage-mode harmonic-reject mixer in $14 \mathrm{~nm}$ FinFET CMOS. In 2017 IEEE international solid-state circuits conference (pp. 220 221).

5. Blanckenstein, J., Klaue, J., Karl, H. (2015): A survey of low-power transceivers and their applications. IEEE Circuits Syst. Mag., 15(3), 6-17.

6. Chakraborty, S., Ivanov, V., Einzinger, J., et al. (2015): An ultra-low power, low-cost multi-standard transceiver. In Texas symposium on wireless and microwave circuits and systems (pp. 1-5)

7. Chiu, C. S., Yen, S. C., Yu, C. Y., Wu, T. H., et al. (2017): 7.3 a $40 \mathrm{~nm}$ low-power transceiver for LTE-A carrier aggregation. In 2017 IEEE international solid-state circuits conference (pp. 130-131).

8. Chuo, L. X., Shi, Y., Luo, Z., Chiotellis, N., et al. (2017): 7.4 A $915 \mathrm{MHz}$ asymmetric radio using Q-enhanced amplifier for a fully integrated $3 \times 3 \times 3 \mathrm{~mm}^{3}$ wireless sensor node with $20 \mathrm{~m}$ non-line-of-sight communication. In 2017 IEEE international solidstate circuits conference (pp. 132-133).

9. Daly, D. C., Chandrakasan, A. P. (2007): An energy-efficient OOK transceiver for wireless sensor networks. IEEE J. Solid-State Circuits, 42(5), 1003-1011.

10. Ericsson (2017): Ericsson mobility report, June 2017. https://www.ericsson.com/ en/mobility-report. August 2017 
11. Fuhrmann, J., Moreira, J., Ossmann, P., Springer, A., Weigel, R., Pretl, H. (2017): A 15-Bit $28 \mathrm{~nm}$ CMOS fully-integrated $1.6 \mathrm{~W}$ digital power amplifier for LTE IoT. In 43th IEEE European solid-state circuits conference (pp. 199-202).

12. Fulde, M., Belitzer, A., Boos, Z., Bruennert, M., Fritzin, J., Geltinger, H., Groinig M., Gruber, D., Gruenberger, S., Hartig, T., Kampus, V., Kapfelsberger, B., Kuttner, F., Leuschner, S., Maletz, T., Menkhoff, A., Moreira, J., Paussa, A., Ponton, D., Pretl, H., Sira, D., Steinacker, U., Stevanovic, N. (2017): 13.2 A digital multimode polar transmitter supporting $40 \mathrm{MHz}$ LTE carrier aggregation in $28 \mathrm{~nm}$ CMOS. In 2017 IEEE international solid-state circuits conference (pp. 218-219)

13. Gebhard, A., Motz, C., Kanumalli R, S., Pretl, H., Huemer, M. (2017): Nonlinear least-mean-square algorithm for second-order interference cancellation in LTE-A RF transceivers. Accepted at Asilomar conference on signals, systems and computers.

14. Horiguchi, F. (2012): Integration of series-connected on-chip solar battery in a triplewell cmos Isi. IEEE Trans. Electron Devices, 59(6), 1580-1584.

15. Jahan, M. S., Langford, J., Holleman, J. (2015): A low-power FSK/OOK transmitter for $915 \mathrm{MHz}$ ISM band. In IEEE radio frequency integrated circuits symposium (pp. 163166).

16. Jones, H. (2014): Whitepaper: strategies in optimizing market positions for semiconductor vendors based on IP leverage. International Business Strategies. Inc. (IBS)

17. Kim, Y., Jang, P., Jin, T., Lee, J., et al. (2017): A current-efficient wideband cellular for multi-band inter- and intra-band carrier aggregation using $14 \mathrm{~nm}$ FinFET CMOS. In IEEE radio frequency integrated circuits symposium (pp. 204-207).

18. Kim, Y. J., Bhamra, H. S., Joseph, J., Irazoqui, P. P. (2015): An ultra-low-power R energy-harvesting transceiver for multiple-node sensor application. IEEE Trans. Circuits Syst. II, Express Briefs, 62(11), 1028-1032.

19. van Langevelde, R., van Elzakker, M., van Goor, D., Termeer, H., Moss, J., Davie, A. (2009): An ultra-low-power 868/915 MHz RF transceiver for wireless sensor network applications. In Radio frequency integrated circuits symposium (pp. 113-116).

20. Law, M. K., Bermak, A. (2010): High-voltage generation with stacked photodiodes in standard CMOS process. IEEE Electron Device Lett., 31(12), 1425-1427.

21. Le, T., Mayaram, K., Fiez, T. (2008): Efficient far-field radio frequency energy harvesting for passively powered sensor networks. IEEE J. Solid-State Circuits, 43(5), 1287-1302.

22. Lu, J., Kovalgin, A. Y., van der Werf, K. H., et al. (2011): Integration of solar cells on top of CMOS chips - Part I: a-Si solar cells. IEEE Trans. Electron Devices, 58(7), 2014-2021.

23. Lu, J., Liu, W., Lu, A. Y., Sun, Y., Lu, J. (2011): Integration of solar cells on top of CMOS chips - Part II: CIGS solar cells. IEEE Trans. Electron Devices, 58(8), 2620-2627.

\section{Authors}

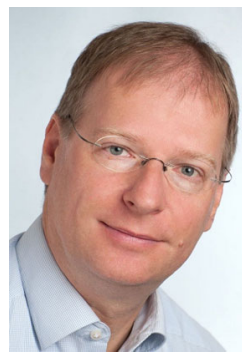

\section{Harald Pretl}

received the Dipl.-Ing. degree in electrical engineering from the Graz University of Technology, Austria, in 1997 and the Dr. techn. (Ph. D.) degree from the Johannes Kepler University (JKU) in Linz, Austria, in 2001. He is a Sr. Principal Engineer with Danube Mobile Communications Engineering $\mathrm{GmbH} \& \mathrm{Co}$ KG (DMCE, majority owned by Intel Corp.) in Linz, Austria, where he has been contributing to multiple generations of cellular RF transceivers and mobile communications platforms as analog circuit designer, project lead and RF systems architect. Since 2015 he has also been full professor at the Institute for Integrated Circuits (IIC) at the JKU, where he is heading the Energy-Efficient Analog Circuits and Systems group. Harald Pretl was a member of the technical program committee (TPC) of the ISSCC in 2010-2012 and has published more than 20 papers at international conferences and journals in the area of RF transceivers, in addition to more than 25 issued and filed patents. His current research interests are focused on highly integrated GSM/UMTS/LTE/5G transceivers, integrated CMOS power amplifiers for mobile communications and loT, wireless sensor networks and low-power RF SoC. Harald Pretl is a member of the IEEE Solid-State Circuits Society and the Austrian Electrotechnical Association (OVE).
24. Milosiu, H., Oehler, F., Eppel, M. (2011): Sub-10 $\mu$ A data reception with low latency using a 180-nm CMOS wake-up receiver at $868 \mathrm{MHz}$. In Semiconductor conference Dresden (SCD) (pp. 1-4).

25. Palazzi, V., Hester, J., Bito, J., Alimenti, F., et al. (2017): A novel ultra-lightweight multiband rectenna on paper for RF energy harvesting in the next generation LTE bands. IEEE Trans. Microw. Theory Tech., 99, 1-14.

26. Pandey, J., Otis, B. P. (2011): A sub-100 $\mu \mathrm{W}$ MICS/ISM band transmitter based on injection-locking and frequency multiplication. IEEE J. Solid-State Circuits, 46(5), 1049-1058

27. Razavi, B. (2004): A study of injection locking and pulling in oscillators. IEEE J. SolidState Circuits, 39(9), 1415-1424.

28. Sadjina, S., Dufrene, K., Kanumalli, R., Huemer, M., Pretl, H. (2017): A circuit technique for blocker-induced modulated spur cancellation in 4G LTE Carrier Aggregation Transceivers. In IEEE Austrochip workshop on microelectronics (pp. 23-27).

29. Schumacher, T., Stadelmayer, M., Faseth, T., Pretl, H. (2017): A review of ultra-lowpower and low-cost transceiver design. In IEEE Austrochip workshop on microelectronics (pp. 29-34)

30. Steffan, C., Greiner, P., Deutschmann, B., Kollegger, C., Holweg, G. (2015): Energy harvesting with on-chip solar cells and integrated DC/DC converter. In 45th European solid state device research conference (pp. 142-145).

31. Vuksan, D. (2017): On-chip photovoltaic cell and micropower DC-DC converter. Master's thesis, Johannes Kepler University Linz.

32. Vyas, R. J., Cook, B. B., Kawahara, Y., Tentzeris, M. M. (2013): E-WEHP: a batteryless embedded sensor-platform wirelessly powered from ambient digital-TV signals. IEEE Trans. Microw. Theory Tech., 61(6), 2491-2505.

33. Wang, A., Zhao, J., Green, M. (1990): $24 \%$ efficient silicon solar cells. Appl. Phys. Lett., 57(6), 602-604.

34. Wong, A. C. W., Kathiresan, G., Chan, C. K. T., Eljamaly, O., et al. (2008): A 1 V wireless transceiver for an ultra-low-power $\mathrm{SoC}$ for biotelemetry applications. IEEE J. Solid-State Circuits, 43(7), 1511-1521

35. Yu, C., Cao, W., Guo, Y., Zhu, A. (2015): Digital compensation for transmitter leakage in non-contiguous carrier aggregation applications with FPGA implementation. IEEE Trans. Microw. Theory Tech., 63(12), 4306-4318.

36. Zoscher, L., Herkess, P., Grosinger, J., Muehlmann, U., Amschl, D., Bösch, W. (2017): A differential threshold voltage compensated RF-DC power converter for RFID tag ICs. In Integrated nonlinear microwave and millimetre-wave circuits workshop (pp. 1-3).

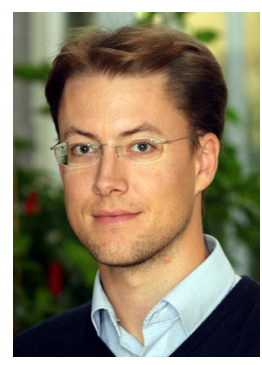

Thomas Faseth

received the Dipl.-Ing. (M.Sc.) and Dr. techn. (Ph.D.) degrees from the Vienna University of Technology, Austria, in the years 2006 and 2012. His career started at the Institute of Electrodynamics, Microwave and Circuit Engineering in the year 2006. Since then he worked in the course of several research projects, especially with the main focus on RFID, localization methods, general RF measurement technology, switched power amplifiers, antenna measurements, as well as material and device characterization. From 2014 till 2016, he was the co-leader of the Microwave Engineering Group at the Institute of Electrodynamics, Microwave and Circuit Engineering at Vienna University of Technology in close cooperation with Asst. Prof. Dr. Holger Arthaber. Since 2016, Thomas Faseth has been with the Institute for Integrated Circuits at Johannes Kepler University Linz and contributes to the Departments of Medical Electronics and Energy-Efficient Circuits and Systems Departments. He is author and co-author of about 30 publications 


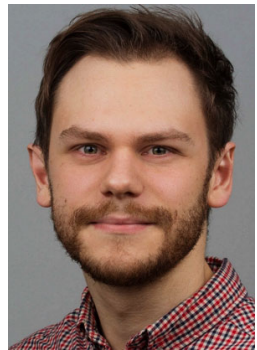

\section{Tim Schumacher}

received the B.Sc. and M.Sc. degrees in electrical engineering from the FriedrichAlexander University Erlangen-Nuernberg, Germany, in 2014 and 2017, respectively. His master thesis addressed the partial design of an integrated pipelined analog-to-digital converter. He joined the Institute for Integrated Circuits (IIC) at the Johannes Kepler University Linz in 2017 as a research assistant and is working towards his Ph.D. degree in electronics and information technology. His current research interests are focused on integrated ultra-low-power receivers.

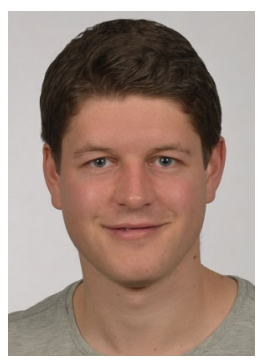

\section{Markus Stadelmayer}

was born in Regensburg, Germany in 1991. $\mathrm{He}$ received the B.Sc. and M.Sc. in electrical, electronic and communication engineering from the Friedrich-Alexander University Erlangen-Nuernberg, Germany, in 2014 and 2017. From 2014 to 2017 he worked as student research assistant at the Institute for electronics Engineering at the FAU ErlangenNuernberg in the field of mixed-signal integrated circuit design. His master thesis, done in cooperation with the eesy-ic $\mathrm{GmbH}$, dealt with the modeling of a pipeline analog-digital converter with calibration algorithm. He is now working towards his Ph.D. degree at the Institute for Integrated Circuits at the Johannes Kepler University Linz. His research focuses on ultra-low-power RF transceiver design.

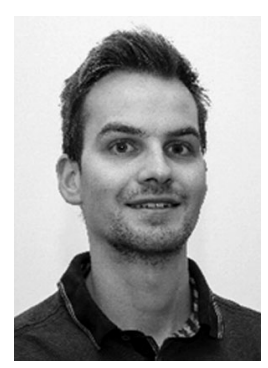

\section{Silvester Sadjina}

was born in Salzburg, Austria in 1988. He received the Bachelor and Master of Science in information electronics from Johannes Kepler University (JKU), Linz, Austria in 2013 and 2015, respectively. In his study he specialized in measurement technnology and communication engineering. In 2014, he was employed as a research assistant at the Institute for Measurement Technology at JKU. Cur- rently, he is working towards the Dr.-Ing. (Ph.D.) degree with the Institute of Integrated Circuits at JKU in cooperation with Danube Mobile Communications Engineering (DMCE) GmbH \& Co KG (majority owned by Intel Austria $\mathrm{GmbH}$ ), Linz, Austria. His research activities focus on the study and development of mixed signal interference cancellation techniques for the mitigation of RX performance degradation in cellular transceiver systems.

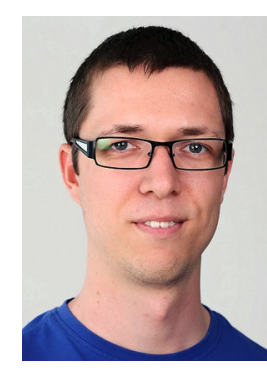

\section{Stefan Schmick}

was born in Linz, Austria in 1990. He received the B.Sc. and M.Sc. degree in electronics and information technology from the Johannes Kepler University (JKU) in Linz, Austria, in 2014 and 2016. His master thesis addressed the design of an integrated currentsteering digital-analog converter. In October 2016 he joined the Institute for Integrated Circuits (IIC) at the Johannes Kepler University Linz working towards his Ph.D. degree. His current research focuses on ultra-low-power RF transceiver design and RF-power-harvesting.

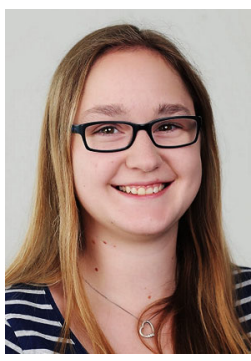

\section{Ehrentraud Hager}

was born in Linz, Austria 1991. She received the B.Sc. and M.Sc. degree in electronics and information technology from the Johannes Kepler University (JKU) in Linz, Austria, in 2015 and 2017. Her master thesis dealt with subthreshold circuit design and low power operational amplifiers. In March 2017 she joined the Institute for Integrated Circuits (IIC) and the Christian Doppler Laboratory for Digitally Assisted RF Transceivers for Future Mobile Communications at the Johannes Kepler University Linz working towards her Ph.D. degree. Her current research focuses on low power circuit design for frequency generation in future transceivers for mobile communications. 